\title{
OPTIMIZING CONTROL OF OPEN BAY ACOUSTICS
}

\author{
A. B. Cain* , A. D. Rubio ${ }^{\dagger}$, D. M. Bortz ${ }^{\ddagger}$, H. T. Banks ${ }^{\S}$, and R. C. Smith ${ }^{\text {I }}$ \\ Center for Research in Scientific Computation \\ North Carolina State University \\ Raleigh, North Carolina
}

\begin{abstract}
$\underline{\text { Abstract }}$
Aircraft with internal carriage of weapons systems require active control strategies to limit high amplitude open bay acoustic resonances and to optimize structure requirements and weapon reliability in an enlarged separation envelope. This paper is focused on communicating an investigation of the use of numerical simulation combined with Proper Orthogonal Decomposition (POD) to optimize an active control system for aircraft weapons bay application. Issues addressed include characterizing shear layer and wake resonant responses, optimal steady blowing rates, the effect of open loop harmonic perturbations, use of POD for post processing data to reduce storage requirements and the use of the Nelder-Mead optimization procedure. Comparison of the wake and shear layer responses shows why no aircraft would ever want to experience a wake response. This study has focused primarily on a freestream flow at $\mathrm{M}=0.85$ with a cavity of aspect ratio $\mathrm{L} / \mathrm{D}=4.5$. The results include the use of steady blowing injection up to $\mathrm{M}=0.9$ and harmonic forcing perturbations ranging in amplitude from $M=0.005$ to $M=0.45$. In the parameter space examined mass injection (displacement effect) has the largest effect and momentum considerations are minor. The best observed forcing reduced the buffet loading metrics by approximately $17 \mathrm{~dB}$.
\end{abstract}

\begin{abstract}
$\underline{\text { Background }}$
Research on flow over an open cavity took a major step forward with the work of Rossiter (1964) who characterized the modal frequency response for subsonic and transonic flows. Since that time many researchers have refined current understanding of the phenomena. This paper is not a comprehensive review but the authors wish to acknowledge the benefit of some of the previous work on prediction and understanding including: fundamental advances by Heller and Bliss (1975), frequency and amplitude prediction by Smith and Shaw (1975), modeling by Tam and Block (1978), fundamentals of shear layer feedback loops by Rockwell (1982), fundamental shear layer behavior by Ho and Huerre (1984), advances to modern aircraft application and control by Shaw $(1982,1998)$, challenges to resonant control of experiments by Gharib (1987), issues on nonlinearity by Keefe (1991), challenges in simulation of resonant control Fuglsang and Cain (1992), the wake/shear layer mode switching studies of Colonius et al. (1999), turbulence model advances implemented by Mani and Ota (1999).
\end{abstract}

Other examples of recent work on cavity acoustics include the papers of Banks et al. (1999), Cain et al. (1999), Cattafesta et al. (1999), Chokani and Lamp (1999), Jacob et al. (1999), Raman et al. (1999),

\footnotetext{
* Associate Fellow AIAA; President, Innovative Technology Applications Company, Chesterfield, MO

Post Doctoral Researcher, presently residing in Argentina

${ }^{\ddagger}$ Ph. D. Candidate, Dept. of Mathematics

${ }^{\S}$ Director of the Center for Research in Scientific Computation and University Professor and Drexel Professor of Mathematics

II Associate Professor of Mathematics and Associate Director of the Center for Research in Scientific Computation

Copyright@2000 The American Institute of Aeronautics and Astronautics Inc. All rights reserved.
} 
Shaw and Northcraft (1999), Stanek et al. (1999), Arunajatesan et al. (2000), and Williams et al. (2000).

\section{$\underline{\text { Approach }}$}

\section{Simulations}

Low dissipation, high accuracy schemes with appropriate data densities are required for efficient simulations that accurately reflect the physical behavior of flow over an open cavity. These explorations have employed third order Runge-Kutta time integration and fifth order spatially refined simulations. To better understand the simulation results, time series, Fourier transforms of time series data, snapshots of vorticity and pressure fields were generated and monitored. The boundary layer turbulence upstream and downstream of the cavity is modeled by the Spalart-Allmaras model using wall functions for efficiency (see Mani and Ota, 1999) and the flow in the cavity region is computed as a direct numerical simulation in two dimensions.

The choice of numerical schemes (third order RungeKutta and fifth order upwind biased) for this work is based on the benchmark problem studies given in Cain and Bower (1995). The present studies have contrasted shear layer mode behavior with wake mode behavior. The wake mode occurs for upstream boundary layers that are very thin relative to the cavity length (see Colonius et al., 1999). Attempts to control the wake mode with forcing met with limited success; this result is consistent with the experiments of Gharib (1987) and the simulations of Fuglsang and Cain (1992), both of which addressed wake mode response. The primary focus of the present investigation is on the shear layer or Rossiter mode response both without forcing and with a search for optimized forcing.

Grid structure, size, forcing and boundary issues The cavity considered was 18 inches long by 4 inches deep. The computational domain extended 30 inches upstream of the cavity and 52 inches downstream of the cavity to help maintain flow quality. Numerical damping was applied locally near the upstream, downstream, and freestream boundaries to limit the influence of reflected waves. The grid is a 3 zone Cartesian grid of approximately 36,000 grid points. The grid was constructed to limit cell to cell size variations. The Reynolds number was reduced by lowering the freestream pressure. This reduction in Reynolds number means that molecular viscosity dominates the numerical dissipation to produce a meaningful physical response. The introduction of mean and harmonic blowing is through a 60 degree angled slot at the upstream corner of the cavity as shown in Fig. 1. This choice of forcing avoids the forcing crossing a zone boundary that may produce non-physical influences.

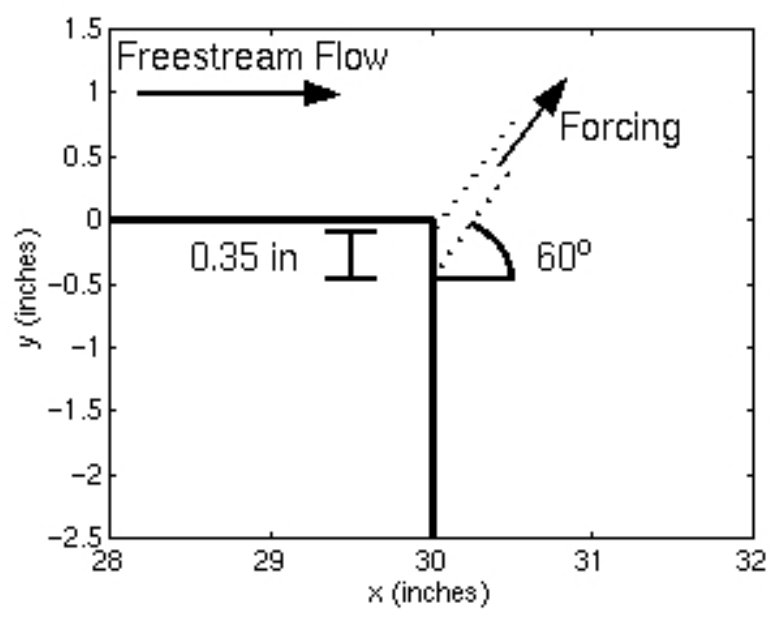

Figure 1 The location of the mean blowing and harmonic blowing slot indicated at the upstream cavity edge.

\section{$\underline{\text { Results }}$}

Characteristics of wake and shear layer modes Figure 2 characterizes the calculated pressure time history for the shear layer and wake modes. The pressure was recorded as a function of time on the downstream cavity wall very near the outer corner as shown in Fig. 3. It is important to notice that the total pressure variation or buffet loading is much greater for the wake mode. Also the average pressure of the wake mode is much greater, resulting in significantly more drag on an air vehicle. Many of the figures in this paper focus on an unsteady pressure or buffet loading measure. This unsteady loading metric is essentially an acoustic source measure. It is thus anticipated that reducing the buffet loading metric will amount to acoustic suppression. Figures 4 and 5 illustrate the significantly different vortical behavior of the shear layer and wake mode responses.

The shear layer mode behavior in Fig. 4 is typical and shows the shear layer bridging the cavity with modest Kelvin-Helmholtz instabilities creating harmonic oscillations in the cavity. In contrast to the shear layer mode, the wake mode response shown in Fig. 5 is characterized by large eruptions of vortical fluid leaving the cavity, and large regions of freestream 
flow impinge on the downstream wall, creating the high pressure signature. A detailed series of snapshots of the vorticity contours of the wake mode evolution shows that the shear layer begins to roll up just inside the cavity. This original concentration of vorticity continues to be fed by the upstream boundary layer until it grows sufficiently large to interact with the downstream wall. This interaction is of such a nature that the large concentration of vorticity is ultimately ejected into the freestream. Colonius et al. (1999) performed high-resolution direct numerical simulations with two-dimensional laminar flow for Reynolds numbers of 30-80, based on the shear layer's momentum thickness. They determined that a criterion predicting wake versus shear layer mode response could be given in terms of the ratio of the thickness of the oncoming shear layer to the cavity length. They observed the switch from shear layer mode to wake mode at ratio of cavity length to momentum thickness of between 50 and 100. In the present studies the momentum thickness Reynolds number of the upstream boundary layer has been varied between 200 and 600 and the switching between shear layer/wake modal responses is consistent with that observed by Colonius et al.

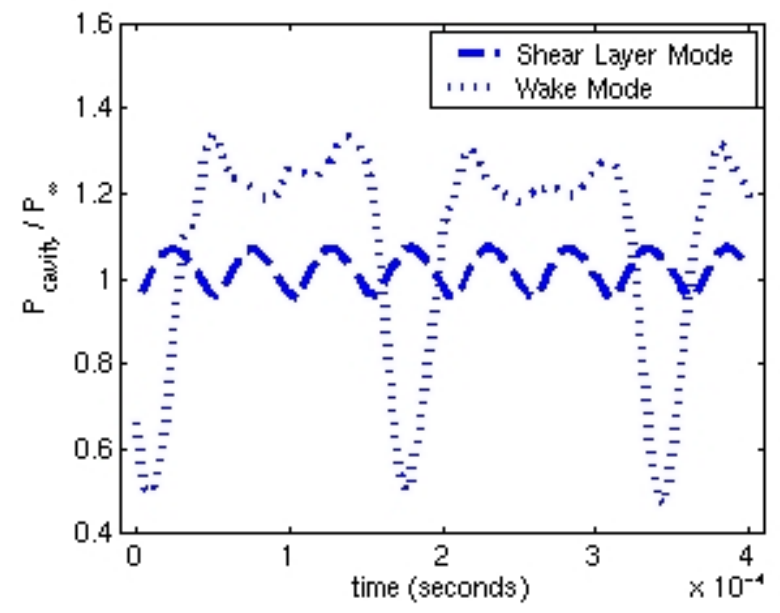

Figure 2 Traces of the time history of pressure on the downstream corner of the wall over several periods of natural oscillations.

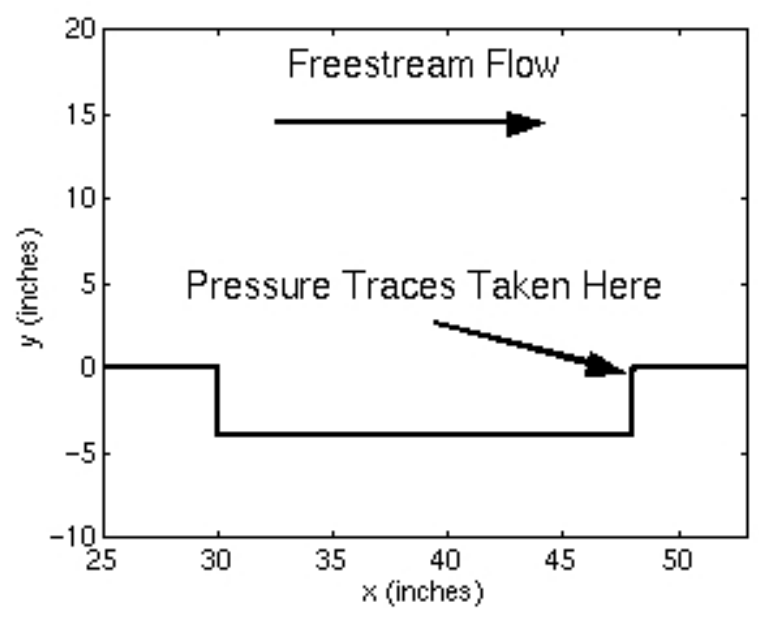

Figure 3 The location of the pressure measurement shown in Figs. 2 and 13.

Forced Cavity Simulations - All simulations considered a cavity of aspect ratio (L/D) 4.5 with a freestream flow of Mach 0.85. A slot at the upstream edge of the cavity, of cross sectional width less than 1/20 of the cavity depth, at an angle of 60 degrees to the oncoming freestream flow, was used to inject mean blowing and unsteady forcing into the cavity boundary layer. Unsteady disturbances composed of a time-mean flow plus a harmonic perturbation of an amplitude up to the level of the mean blowing were studied. The injected blowing disturbances had velocity amplitudes from Mach 0.005 to 0.9 . To assess the effect of forcing on cavity unsteadiness, the integral over the solid boundaries of the square of the difference between the time mean pressure and the instantaneous pressure was used to form metrics of the buffet loading on the front wall, back wall, and the cavity floor (or ceiling). An overview of the cases examined is shown in Fig. 6. The case of no forcing is shown to provide a reference point for the forcing. These cases 


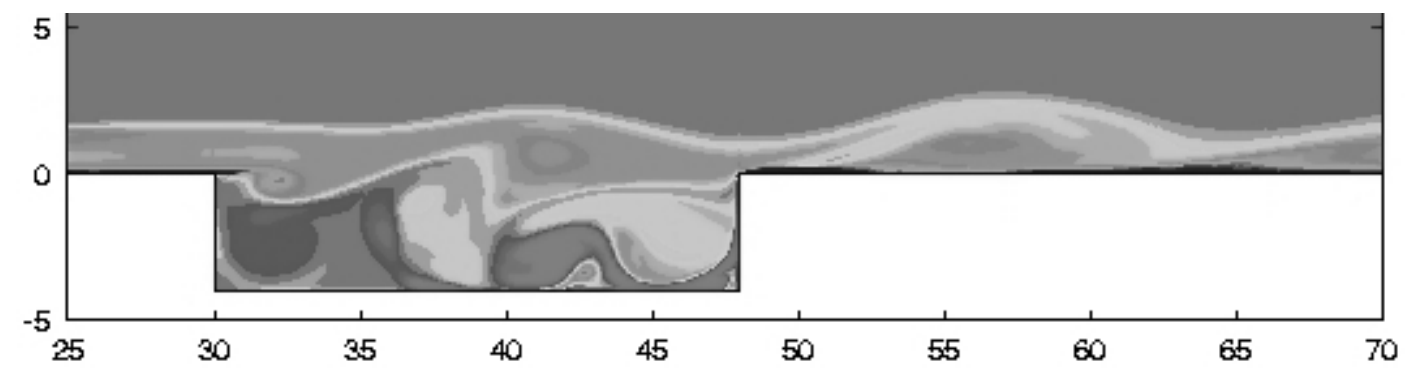

Figure 4 Vorticity contours in a snapshot of the fifth order spatial simulation of the shear layer mode behavior.

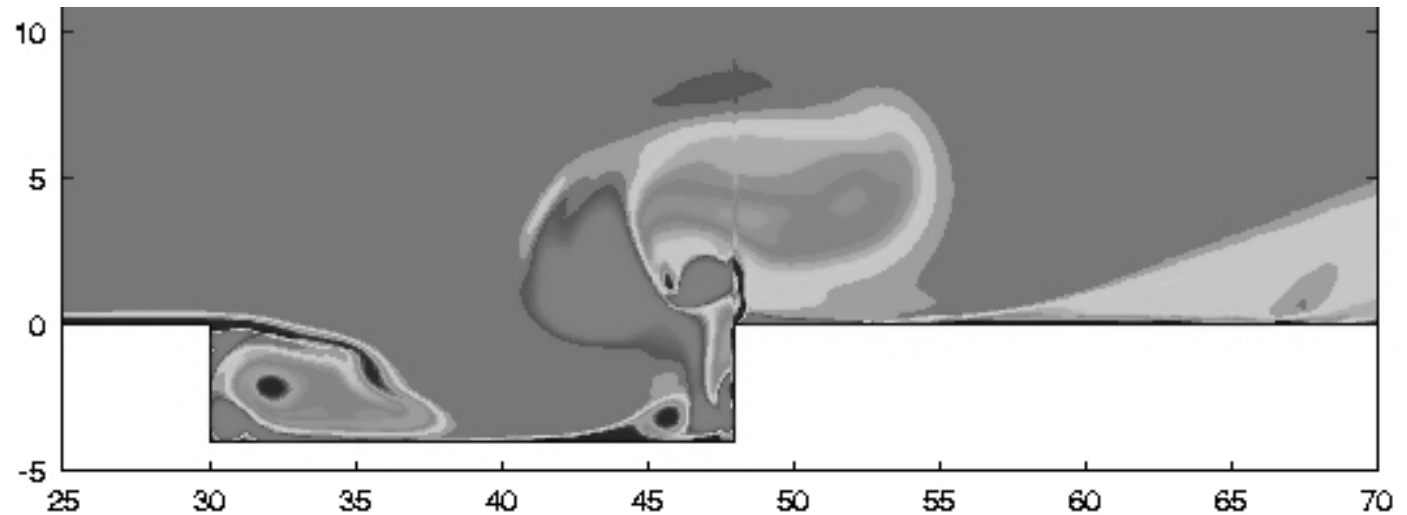

Figure 5 Vorticity contours in a snapshot of the fifth order spatial simulation of the wake mode behavior.

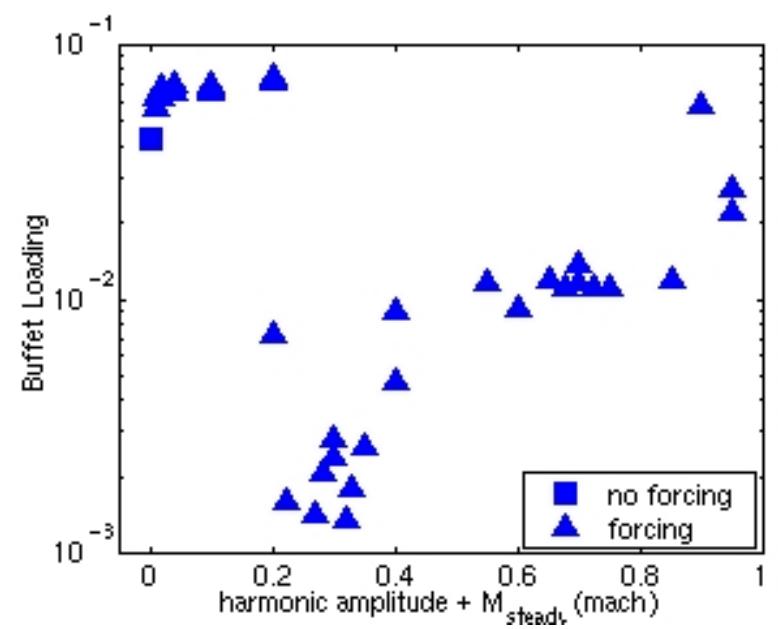

Figure 6 A simple presentation of the cases examined with buffet loading expressed as a function of the sum of mean and harmonic forcing Mach number. include a range of frequencies and the ratio of harmonic amplitude to the mean blowing level is also varied. Note that use of forcing disturbances less than Mach $=0.2$, for the computational configuration examined here, increases the buffet loading but higher values can reduce the buffet loading considerably.

The fact that we were not able to reduce the buffet loading by using small amplitude harmonic blowing suggests that open loop control with small amplitude disturbances is unlikely. The rise in buffet loading at blowing values of Mach $=0.7$ (though not presented in this plot Mach $=0.9$ also displays an upward trend) is believed to be due to the effect of strong entrainment flows that occur at high blowing rates. There may also be an effect of boundary layer separation upstream of the cavity edge. Later figures will show that modest blowing gently lifts the shear layer over the downstream cavity corner and softens the interactions of shear layer vortical instabilities with the corner. 
Figure 7 isolates the effect of mean blowing level on the buffet loading level by fixing the harmonic perturbation level at $\mathrm{M}=0.25$ with a frequency of 361 $\mathrm{Hz}$ (90\% of the second Rossiter mode) and then varying the mean blowing rate. It is readily apparent that there is a flat trough for a mean blowing level ranging from $M=0.3$ to $M=0.6$. It is believed that the level trough is due to the harmonic forcing influence and that the level may come down with lower harmonic amplitudes. Figure 8 shows various harmonic forcing levels for 3 mean blowing levels, all with harmonic frequency of $361 \mathrm{~Hz}$. The loading metric decreases as harmonic levels are decreased until, at small harmonic levels the loading increases as the harmonic forcing goes to zero. Many of the experimental studies to date have focused on the issue of different forcing frequencies. These simulations exhibit very important significant differences with respect to experiments but provide some complementary information on open loop forcing. All cases in Fig. 9 have harmonic blowing amplitude equal to the mean blowing level. The blowing levels range from $\mathrm{M}=0.005$ to $\mathrm{M}=0.45$.

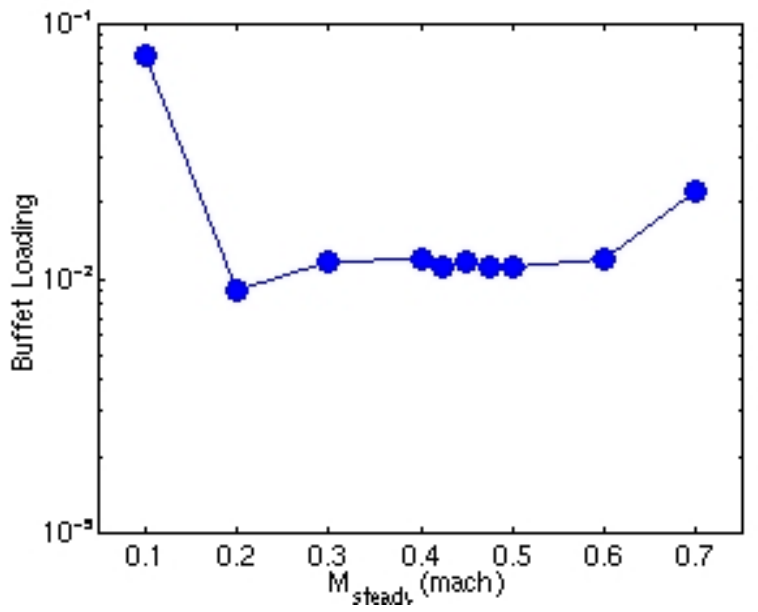

Figure 7 Buffet loading metric as a function of the mean blowing level for a harmonic forcing level of $\mathrm{M}=0.25$ at a frequency of $90 \%$ of the second Rossiter frequency $(361 \mathrm{~Hz})$.

In Fig. 9 a substantial range of frequencies of harmonic excitation shows limited improvement in the buffet loading for these combinations of forcing. The relevance of the frequencies examined, is given a context by the indicated first two Rossiter frequencies. Figure 10 illustrates the behavior of a given harmonic frequency over a range of amplitudes of harmonic forcing and mean blowing. Note that in all cases in Fig. 10 the mean blowing Mach number is equal to the harmonic perturbation amplitude.

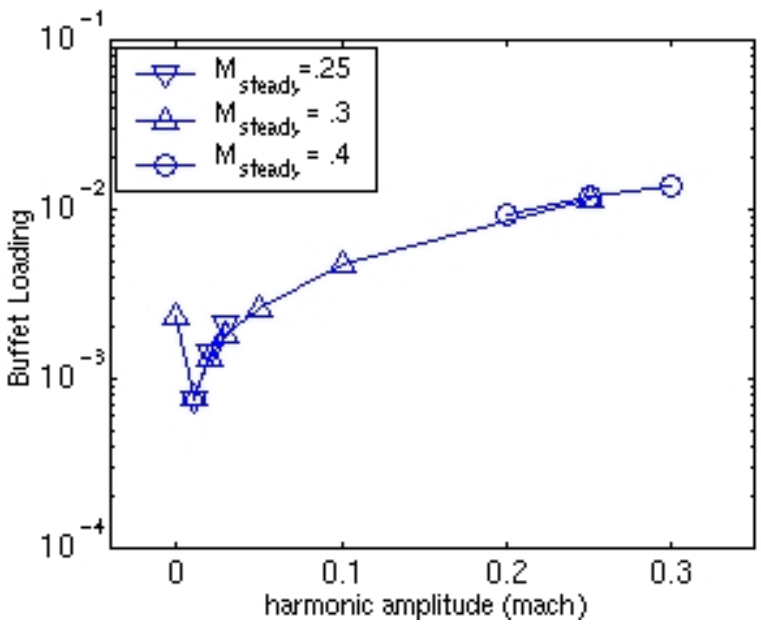

Figure 8 Behavior of the buffet loading metric at several harmonic forcing amplitudes for mean blowing levels of $\mathrm{M}=0.3$ and $\mathrm{M}=0.4$ and frequency of Fig. 7.

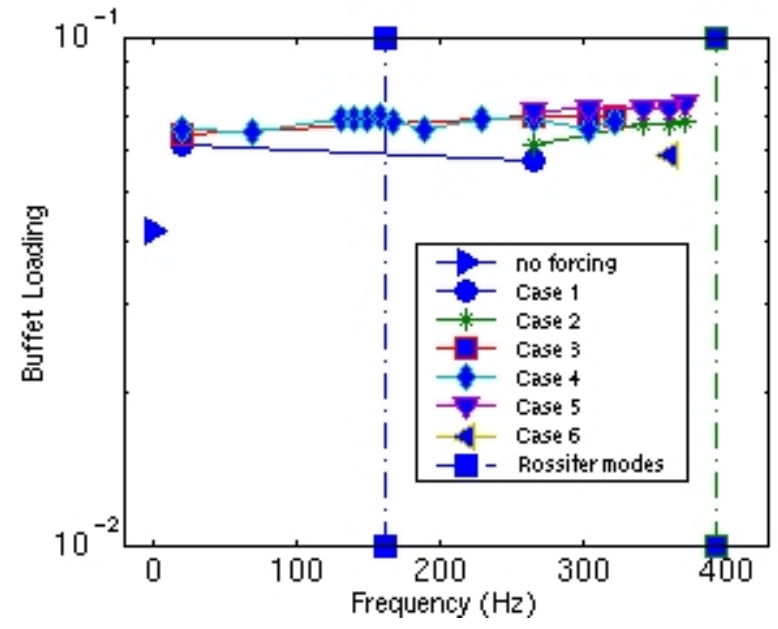

Figure 9 Buffet loading level at many frequencies with the harmonic forcing level equal to the mean blowing for Case 1, M= 0.005; Case 2, $\mathrm{M}=0.01$; Case 3, $\mathrm{M}=0.02$; Case 4, $\mathrm{M}=0.05$; Case 5, $\mathrm{M}=0.1$; and Case 6, M=0.45. 


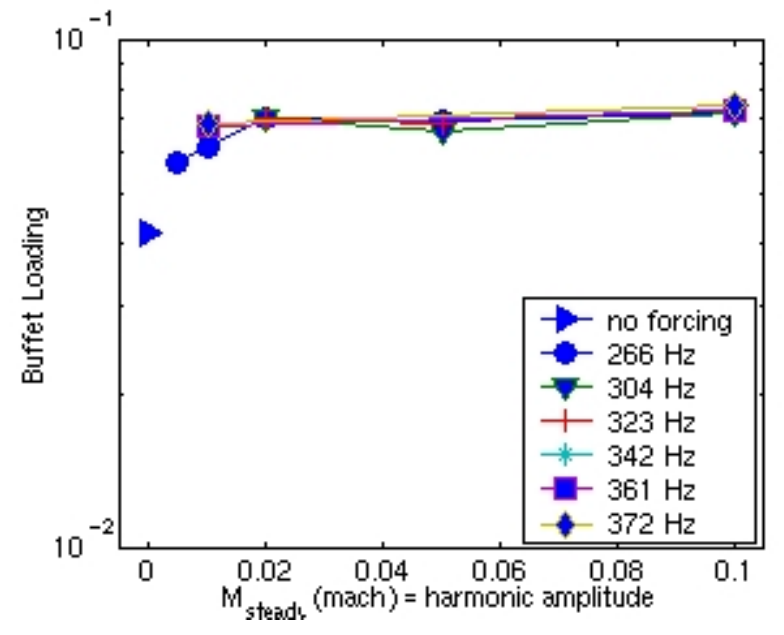

Figure 10 Buffet loading metric for several frequencies with harmonic forcing levels equal to the mean blowing Mach number.
The behavior illustrated in Figs. 6 through 10 suggests that open loop harmonic forcing is limited in reducing the buffet loading in these simple twodimensional numerical simulations. To gain a better understanding of the benefit of the mean blowing we turn to the visualization in Figs. 11 and 12. The conditions for Figure 11 are for an amplitude of harmonic forcing and mean blowing of Mach 0.01. The results are very similar to those for no forcing except that the disturbances are regularized instead of random. Figure 12 shows reduced buffet loading levels relative to the unforced simulations.

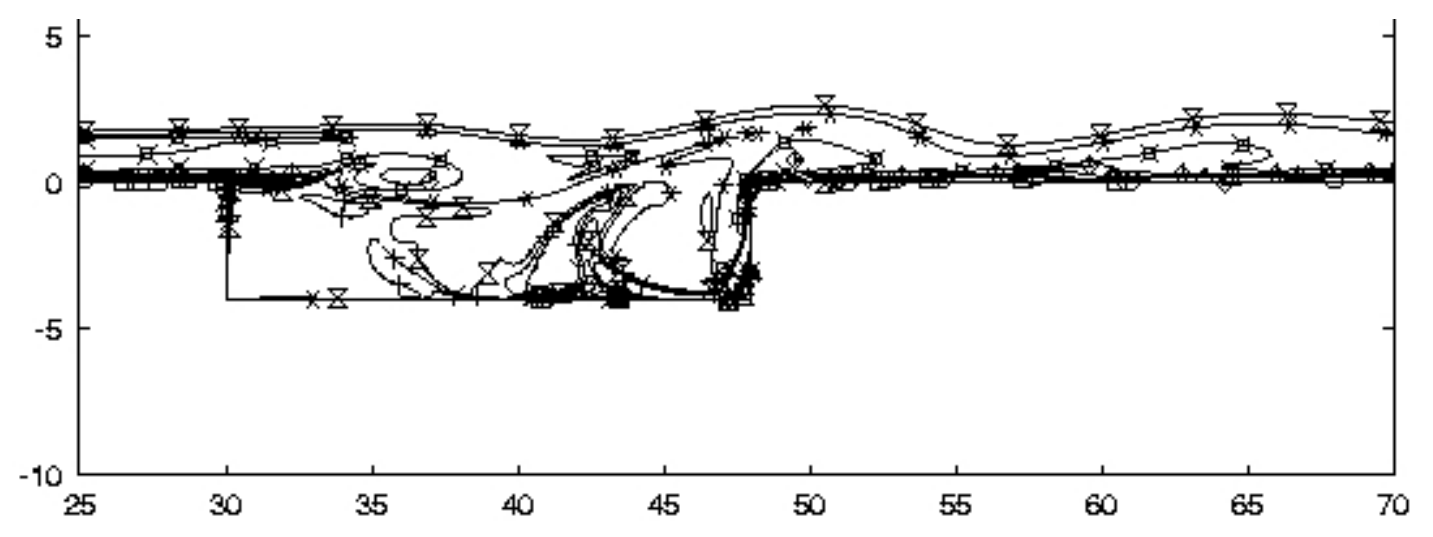

Figure 11 Contours of vorticity for a very weakly forced flow. The freestream Mach number is $\mathrm{M}=0.85$. The mean blowing and harmonic forcing amplitudes are both $\mathrm{M}=0.01$ and the frequency of forcing is $90 \%$ of the second Rossiter frequency $(361 \mathrm{~Hz})$. The contours levels are in increments of vorticity jumps of 2000/sec.. 


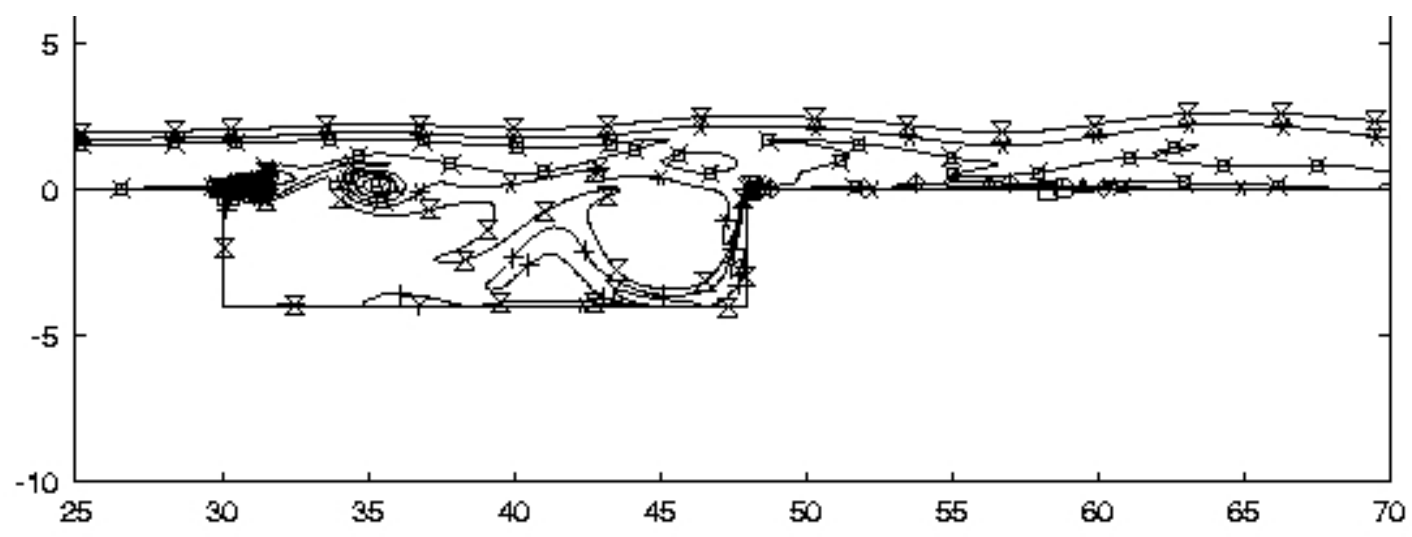

Figure 12 Contours of vorticity for a beneficially forced flow. The freestream Mach number is $\mathrm{M}=0.85$. The mean blowing level is $\mathrm{M}=0.4$ and harmonic forcing amplitude is $\mathrm{M}=0.2$ and the frequency of forcing is $90 \%$ of the second Rossiter frequency $(361 \mathrm{~Hz})$. The contour levels are in increments of vorticity jumps of 2000/sec..

The acoustic suppression was generated by mean blowing at a Mach number of 0.4 with harmonic forcing frequency at $90 \%$ of the second Rossiter mode frequency at an amplitude of Mach 0.2. Both of these simulations show the forcing-induced development of the Kelvin-Helmholtz instability. In the case of the suppressed case, however, the mean blowing gently raises the perturbed shear layer over the downstream corner and the vortical interactions with the corner are reduced. Another aspect of this effect is illustrated by the stronger gradients of vorticity inside the cavity in Fig. 11 indicating strong shear layers and the presence of more dynamic activity in the cavity. Figure 13 shows the reduced buffet loading (pressure time history) compared to shear layer and wake modes with no blowing.

Proper Orthogonal Decomposition To simplify the post processing of the numerical simulation data we take advantage of the Proper Orthogonal Decomposition (POD). The Proper Orthogonality Decomposition (POD) is a method for representing the elements of a vector field in an $L^{2}$ optimal sense. In this case, the vector field is a set of time-dependent pressure values at each grid point in our domain. We will use POD to represent the pressure as a linear combination of a finite set of basis vectors. This combination takes the form:

$$
\begin{aligned}
& p(t, x)=\sum_{i=1}^{K} a_{i}(t) \phi_{i}(x) \\
& =\sum_{i=1}^{K}\left\langle p(t, x), \phi_{i}(x)\right\rangle_{L^{2}} \phi_{i}(x)
\end{aligned}
$$

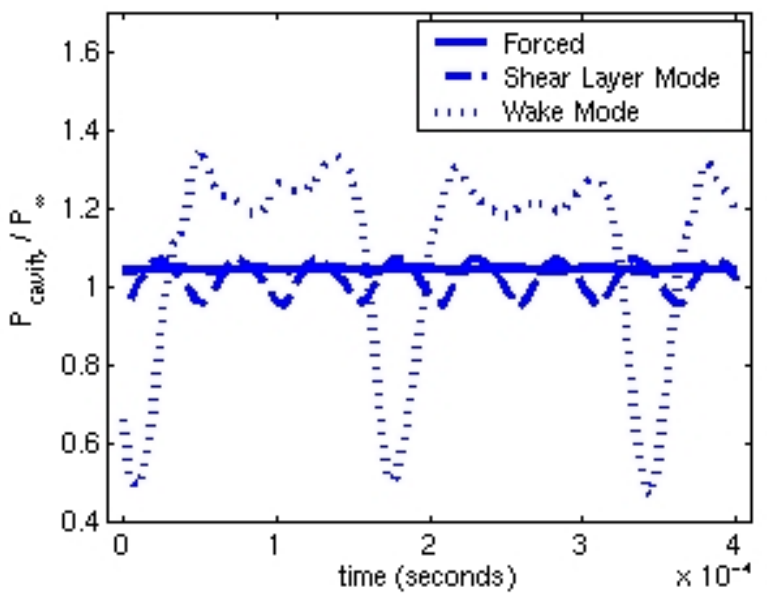

Figure 13 The buffet loading metrics for the wake mode (most extreme), the unforced shear layer response, and the beneficially forced shear layer.

The POD method is a constructive approach to determining these basis elements in an efficient manner using the time-dependent snapshots $p_{j}(x)=p\left(t_{j}, x\right)$. We begin by supposing that the elements in the basis are of the form $\phi_{i}(x)=\sum_{j=1}^{N} c_{i j} p_{j}(x), i=1, \ldots, K$.

Following a standard method, (for details see Ly andTran,1998, Banks, del Rosario and Smith, 1999) we find that the calculation of the bases reduces to an 
eigenvalue problem of the form $\left[\left\langle p_{i}(x), p_{j}(x)\right\rangle\right] v_{k}=\lambda_{k} v_{k}$, with each of the $p_{j}$ 's being a vector of pressures at a specific time $t_{j}$ and $\left[\left\langle p_{i}(x), p_{j}(x)\right\rangle\right]$ being a matrix whose $(i, j)$ th element is $\left\langle p_{i}(x), p_{j}(x)\right\rangle$. The coefficients $c_{i j}$ in the basis representation are then obtained from the eigenvectors $v_{k}$ in a straightforward manner.

It is possible to show that if the vector field ( $p$ in this case) is in units of velocity, we can nondimensionalize the eigenvectors, thus giving the eigenvalues units of velocity. Based on results from [Riesz, Sz-Nagy] we know that if we choose $n$ large enough: $\sum_{i=1}^{n} \lambda^{2}{ }_{i} \approx \sum_{i=1}^{N} \lambda^{2}{ }_{i}<\infty$. Therefore, it is theoretically possible to choose a reduced number $n$ and capture a significant amount of the 'total energy' (of the system represented in the snapshots) using only $n$ bases. We preformed a POD decomposition on a set of pressure field time-dependent snapshots of systems that exhibited Rossiter and wake mode responses (with and without forcing). Figure 14 depicts three representative cases where the magnitude of the 'energy' in a given POD element drops off rapidly as we increase the mode number. Indeed, as Figure 15 reveals, we can capture more than $99.999 \%$ of the 'total energy' in 100 snapshots using only 4 of the POD basis elements.

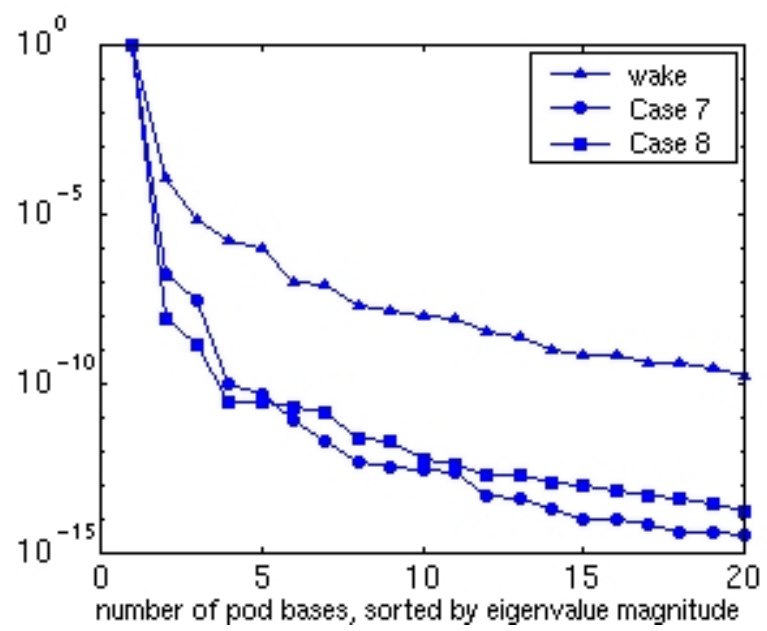

Figure 14 Distribution of amplitudes of POD elements. The wake mode is that referred to in Fig. 2 , as is Case 7 which is the natural Shear Layer response. Case 8 is a forced response that substantially reduced the buffet metric.

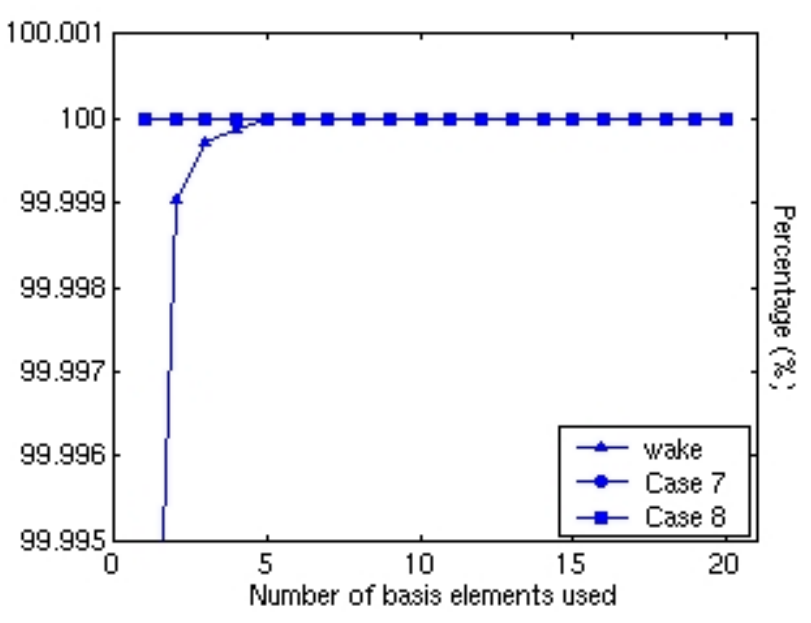

Figure 15 Measure of POD convergence based on relative 'energy' of pressure representation. See caption 14.

In using WIND to run our simulations, each spatially discretized snapshot requires approximately $2 \mathrm{MB}$ of storage in an ASCII format. Thus to store $\mathrm{N}=100$ snapshots would require $200 \mathrm{MB}$. Projecting onto the first 10 POD basis elements, these elements and the coefficients for 100 snapshots require only $2 \mathrm{MB}$ of storage. Clearly POD is an extraordinarily valuable tool for reducing storage requirements in carrying out simulations and control calculations. We are aware that simply compressing the ASCII data would also save considerable space and present these results to describe the relative merits of POD in the absence of other storage saving mechanisms.

In an attempt to reduce RMS pressure metrics (analogous to buffet loading), we injected air (at the upstream edge of the cavity) at a variety of frequencies, steady blowing amplitudes, and unsteady blowing amplitudes. For each set of forcing parameters we have calculated a metric from a sum of squares of the difference between the instantaneous pressure and the average pressure. Let $D$ be a specific sub-domain of the spatial grid (e.g., the grid points along the downstream wall of the cavity), $d$ be the length of $D$, and let $\bar{p}(x)$ be the time-average pressure at the point $\mathrm{x}$. Therefore, our metric is of the form $J=\frac{1}{p_{\infty}}\left\{\frac{1}{N d} \sum_{j=1}^{N} \int_{x_{k} \in D}\left|p\left(t_{j}, x_{k}\right)-\bar{p}\left(x_{k}\right)\right|^{2} d x\right\}^{1 / 2}$,

where we used the trapezoid method to approximate the integral. We calculated the metric using three distinct sub-domains: the downstream wall, the 
upstream wall, and the bottom wall (all in the cavity). We found that each of the three metrics were, in general, directly proportional to one another, and thus focused our attention on the downstream wall metric. Using a polynomial interpolation over the parameter space, we then used Nelder-Mead in an attempt to minimize the downstream metric over the parameter space. We chose Nelder-Mead because the algorithm does not require the calculation of gradients and tends to work well when optimizing over a low dimensional space.

Comparing numerical simulations to experimental data While numerical simulations are of considerable value for sorting out hypotheses, it is essential to realize that there are potentially very significant differences between the assumptions used in numerical simulations and laboratory or flight situations. The first major simplification is that of two-dimensional simulation. While simulations can be performed which include some aspects of threedimensionality, a parametric study in three dimensions was computationally prohibitive, given our computational resources. Any simulations of the cavity that include full three-dimensional simulation of the turbulence in the oncoming boundary layer are also computationally prohibitative.

\section{$\underline{\text { Discussion }}$}

These computational studies were done in the context of a numerically focused study in two dimensions without guidance from experiments. Smith (2000) and Shaw (2000) have both pointed out that cavity simulations performed in three-dimensions often behave significantly different than two-dimensional simulations. One manifestation of this approach was that a large number of forcing conditions were examined before finding a benefit of forcing. It is interesting to learn that in early experimental studies Shaw (2000) examined many forcing conditions finding substantial numbers of cases both with beneficial results and without beneficial results.

Comparison of Simulations with the Experiments of Leonard Shaw

These simulations were done without knowledge of the parameter space used in Shaw's experiments. As a result, the cavity geometry, blowing geometry, and blowing velocities are quite different in the experiments than in the simulations. In an attempt to compare the experimental and simulation results, a simple engineering forcing parameter is proposed.
The parameter is based on the volumetric inflow of the forcing stream per unit width, the freestream velocity and the cavity length. The parameter is:

\section{$\chi=$ Vin $*$ Gslot/(Uinf*Lcavity)}

where Vin is the vertical component of the blowing velocity, Gslot is the effective blowing slot gap, Uinf is the freestream velocity, and Lcavity is the length of the cavity. In the case of the experimental information used here, the blowing flow chokes and the fully expanded blowing velocity was used to compute $\chi$. The experimental values shown below are those from data provided by Shaw (2000).

$\underline{\text { Table of blowing parameter values }}$

\begin{tabular}{|c|c|c|}
\hline Data Type & $\begin{array}{c}\text { Mass Flow/ } \\
\text { Mach } \\
\text { injection }\end{array}$ & $\chi$ \\
\hline Experimental A & $0.3 \mathrm{lbs} / \mathrm{sec}$. & 0.0012 \\
\hline Experimental A & $0.4 \mathrm{lbs} / \mathrm{sec}$. & 0.0016 \\
\hline Experimental A & $0.5 \mathrm{lbs} / \mathrm{sec}$. & 0.0020 \\
\hline Experimental B & $0.3 \mathrm{lbs} / \mathrm{sec}$. & 0.0012 \\
\hline Experimental B & $0.4 \mathrm{lbs} / \mathrm{sec}$. & 0.0016 \\
\hline Experimental B & $0.5 \mathrm{lbs} / \mathrm{sec}$. & 0.0020 \\
\hline Computational & Mach 0.2 & 0.0013 \\
\hline Computational & Mach 0.3 & 0.0020 \\
\hline Computational & Mach 0.4 & 0.0026 \\
\hline Computational & Mach 0.5 & 0.0033 \\
\hline Computational & Mach 0.6 & 0.0040 \\
\hline Computational & Mach 0.7 & 0.0046 \\
\hline
\end{tabular}

Mass effects versus momentum effects The fact that the mass flux weighted parameter range matches for the experiments and computations supports the idea that the primary suppression effect derives from the displacement of the shear layer. This is particularly significant since the momentum parameter is 3-15 times larger for the experiment than it is in the computational cases. It is believed that the experiment did not benefit from forcing in the horizontal injection cases because it essentially amounts to placing an acoustic source inside the cavity. Recall that the simulations seem to indicate that the harmonic forcing at large amplitudes drives the buffet metric (see Fig. 7). The experimental harmonic velocities are 3-15 times the computational levels but may not produce the same impact since the geometries are usually quite different. 


\section{$\underline{\text { Conclusions }}$}

1. Shear layer and wake mode responses have been demonstrated computationally. Wake modes are very undesirable and understanding them better will help to insure no aircraft designs in the wake mode space are considered.

2. Steady blowing can successfully reduce buffeting metrics. Blowing level has an optimal value. The present computations and experiments of Shaw seem to be consistent in the level of blowing that has a beneficial impact. High blowing levels can create an entrainment induced cavity disturbance.

3. A simple algebraic parameter has been defined. This parameter seems to relate disparate experiments and computations and provide guidance for optimal open loop control.

4. In addition to steady blowing, the effect of harmonic excitation was examined. Many initial cases at many frequencies and amplitudes showed no benefit from harmonic excitation. However, eventually we found that small amplitude harmonic forcing in addition to a mean component of blowing provided the minimum buffet metric observed in the present investigation.

5. Proper Orthogonal Decomposition provides a valuable tool for post processing. It reduces computation and more importantly reduces storage requirements.

6. POD can be incorporated in optimization schemes such as Nelder-Mead to accelerate finding an optimal solution.

\section{Acknowledgements}

The authors wish to acknowledge the benefit of discussions with Tim Colonius, Ed Kerschen, Val Kibens, Mori Mani, Leonard Shaw, and Dave Williams. North Carolina State University also wishes to acknowledge the software support of The Boeing Company. An essential element that made this work possible was the support from AFOSR under the program management of Marc Jacobs through the grant under AFOSR-F49620-98-1-0180.

\section{$\underline{\text { References }}$}

S. Arunajatesan, N. Sinha, and S. Menon, "Towards Hybrid LES-RANS Computations of Cavity Flowfields," AIAA-2000-0401, January 2000.

H. T. Banks, R.C.H. del Rosario, and R. C. Smith, Reduced Order Model Feedback Control Design: Numerical Implementation in a Thin Shell Model, CRSC-TR98-27, July, 1998, N. C. State Univ.; IEEE Transactions Auto. Control, to appear.

Banks, H., Rubio, D., and Smith, R., "Modeling Acoustic Fields Generated by Flow Past an Open Cavity," AIAA-99-1913, May 1999.

Cain, A. B., Bower, W. W., and McCotter, F., "Quick Turnaround Prediction of Weapons Bay Cavity Acoustic Resonance," AIAA-99-1899, May 1999.

Cain, A. B. and Bower, W. W., "Comparison of Spatial /numerical Operators for Duct-Nozzle Acoustics," Proceedings of the ICASE/LaRC Workshop on Bench Mark Problems in Computational Aeroacoustics, NASA Conference Publication 3300, May 1995.

Cattafestta, L. III, Shukla, D., Garg, S., and Ross, J., "Development of an Adaptive Weapons-Bay Suppression System," AIAA-99-1901, May 1999.

Chokani, N. and Lamp, A., "Computation of Unsteady Incompressible Cavity Flow with Pulsed Jet Control,” AIAA-99-1914, May 1999.

Colonius, T., Basu, A. and Rowley, C., "Numerical Investigation of Flow Past a Cavity," AIAA-99-1912, May 1999.

Colonius, T., Private communication, September 1999.

Fuglsang, D. F. and Cain, A. B., "Evaluation of Shear Layer Cavity Resonance Mechanisms by Numerical Simulation,” AIAA 92-0555, January, 1992.

Gharib, M., "Response of the Cavity Shear Layer Oscillations to External Forcing," AIAA Journal, Vol. 25, No. 1, pp. 43-47, 1987.

Heller, H. H. and Bliss, D. B., "The Physical Mechanism of Flow Induced Pressure Fluctuations in Cavities and Concepts for Suppression," AIAA $2^{\text {nd }}$ Aeroacoustics Conference, Paper 75-491, March 1975. 
Ho, C. M. and Huerre, P., "Perturbed Free Shear Layers," Annual Review of Fluid Mechanics, Vol. 16, 1984, pp. 365-424.

Jacob, M., Gradoz, V., Louisot, A., Juve, D., Guerrand, S., "Comparison of Sound Radiated by Shallow Cavities and Backward Facing Steps," AIAA-99-1892, May 1999.

Keefe, L., "Multiple Solutions. A Barrier to Flow Control?," Presented at the Forty-Fourth Annual Meeting of the Division of Fluid Dynamics of the American Physical Society, Scottsdale AZ, Nov. 1991.

H. V. Ly and H. T. Tran, Proper Orthogonality Decomposition for Flow Calculations and Optimal Control in a Horizontal CVD Reactor, CRSC-TR9813, March 1998, N. C. State Univ.; Quarterly of Applied Mathematics to appear.

Mani, M. and Ota, D., “A Compressible Wall Function for Steady and Unsteady Flow Applications," AIAA 99-3216, June 1999.

J. A. Nelder and R. Mead, A Simplex Method for Function Minimization,

Comput. J., 7(1965), pp. 308-313.

Raman, G., Raghu, S., and Beninc, T., " Cavity Resonance Suppression Using Minature Fluidic Oscillators," AIAA-99-1900, May 1999.

Rockwell, D., "Invited Lecture: Oscillations of Impinging Shear Layers," AIAA 82-0047, January 1982.
Rossiter, J. E., "Wind Tunnel Experiments on the Flow Over Rectangular Cavities at Subsonic and Transonic Speeds," Royal Aircraft Establishment, TR No. 64307, October 1964.

Shaw, L. L., "Full Scale Flight Evaluation of Suppression Concepts for Flow-Induced Fluctuating Pressues in Cavities,” AIAA 82-0329, January 1982.

Shaw, L. L., "Active Control for Cavity Acoustics," AIAA 98-2347.

Shaw, L., and Northcraft, S., "Closed Loop Active Control for Cavity Acoustics," AIAA-99-1902, May 1999.

Shaw, L, private communication, March 2000.

Smith, B. private communication, January 2000.

Smith, D. L. and Shaw, L. L., "Prediction of the Pressure Oscillations in Cavities Exposed to Aerodynamic Flow," AFFDL-TR-75-34, October 1975.

Stanek, M., Sinha, R., and Birkbeck, R., "AcousticsCompatible Active Flow Control for Optimal Weapons Separation,” AIAA-99-1911, May 1999.

Tam, C. K. W. and Block, P. J. W., "On the Tones and Pressure Oscillations Induced by Flow over Rectangular Cavities," Journal of Fluid Mechanics, Vol. 89. Part 2, pp. 373-399, 1978.

Williams, D. R., Fabris, D., Iwanski, K., and Morrow, J., "Closed-Loop Control in Cavities with Unsteady Bleed Forcing," AIAA-2000-0470, January 2000 . 